\title{
Estructura de la comunidad bentónica (infaunal) en el delta del Río Colorado, México
}

\author{
Guillermo Villarreal Chávez ${ }^{1}$ y Carlos Melecio Carmona Cedillo 2
}

1 Facultad de Ciencias Marinas, Universidad Autónoma de Baja California. Apdo Postal 453, Ensenada, Baja California, México. 22880. Fax 61744103. Correo electrónico gvc@bahia.ens.uabc.mx

2 Centro de Investigaciones Avanzadas, Instituto Politécnico Nacional, Mérida, Yucatán, México.

Recibido 24-II-1999. Corregido 10-VI-1999. Aceptado 23-VI-1999.

\begin{abstract}
The benthic community structure of the Colorado River delta, Mexico, was studied by digging cores in six points along a line perpendicular to the coastline. Three communities were found. The external community is influenced by human activities, but the community indexes are similar to others reported in the literature for equivalent areas. The middle community has the highest density and biomass in the area, apparently because of bioturbation created by the crab Uca princeps. The internal community is poor in density and biomass, possibly because of the instability caused by erosion related to the tide flux.
\end{abstract}

Key words: Macrobenthos, communities, Colorado River delta, Mexico.

El estuario del Río Colorado ha cobrado importancia científica en los últimos años, sobre todo a partir de la declaratoria de la zona como Reserva de la Biosfera en 1994. Como consecuencia se han realizado estudios para comprender el funcionamiento ecológico del área. De éstos se ha derivado una imagen de la zona como un centro de gran producción orgánica con altas biomasas de invertebrados (Farfán y Alvarez-Borrego 1992, VillarrealChávez 1992).

Sin embargo el Delta del Río Colorado ha sufrido un fuerte impacto humano relacionado con la construcción de represas entre 1938 y 1963. Esto ha provocado, un bloqueo absoluto del flujo de agua dulce, y el establecimiento de un estuario euhalino que presenta salinidad entre 33 y $37 \%$ o en toda su extensión. (Martínez-Rojas-Reynoso 1990).

En este trabajo se aborda la descripción y análisis de la comunidad bentónica (infaunal) del delta como base para futuros estudios, especialmente sobre cambios en el tiempo.

\section{MATERIALES Y MÉTODOS}

Se trabajó en la zona intermareal de la desembocadura del Río Colorado en seis puntos sobre la costa oriental del Golfo de 
California (entre $31^{\circ} 43^{\prime} 32^{\prime \prime}$ y $31^{\circ} 45^{\prime} 28^{\prime \prime} \mathrm{N}$; $114^{\circ} 43^{\prime} 12^{\prime \prime}$ y $114^{\circ} 28^{\prime} 38^{\prime \prime} \mathrm{W}$ ). Los seis puntos se denominan en el trabajo: CPN, localizada a $5 \mathrm{~km}$ al sur del pueblo de Golfo de Santa Clara, SCLA, en la playa adyacente al Santa Clara; ELFB, a cinco $\mathrm{km}$ al norte del pueblo; ESBA, a tres $\mathrm{km}$ al norte de la anterior; ILGOR, a tres $\mathrm{km}$ al norte de ESBA; e ILMON, a cinco $\mathrm{km}$ al norte de la anterior.

En cada una de las estaciones se tomaron cuatro muestras separadas $25 \mathrm{~m}$ entre sí siguiendo un transecto perpendicular a la línea de costa. Cada muestra cubre un área de $0.025 \mathrm{~m}^{2}$. La separación se realizó con tamices de 0.5 $\mathrm{mm}$ de luz de malla. El análisis granulométrico se realizó por el método de Folk (1961), en dos muestras de sedimento superficial de cada estación. La concentración de materia orgánica se midió por el método de ignición de Dean (1974). Se determinó un índice de valor de importancia (ivi) siguiendo el método usado por Brian y Curtis (1952).

\section{RESULTADOS}

Los resultados del análisis de escalamiento multidimensional (MDS) muestran tres grupos de estaciones. El primer grupo está formado por las estaciones ECPN y SCLA (comunidad externa); el segundo por ELFB, ESBA e
ILGOR (comunidad media), y el tercero por ILMON, (comunidad interna). La separación muestra un eje que va desde las estaciones externas al estuario, con sedimento arenoso hasta las internas con sedimento limoso y limo-arcilloso.

La comunidad externa se caracteriza por un sedimento predominantemente arenoso, con porcentaje promedio de materia orgánica de $0.66 \pm 0.004$. Presenta un valor de riqueza de especies de 14, con una densidad media de organismos de $36.2 \pm 12.4 \mathrm{inds} / \mathrm{m}^{2}$ y con una biomasa media de $2.04 \pm 1.8 \mathrm{mg} / \mathrm{m}^{2}$. La diversidad de Shannon-Weaver (H') en esta comunidad se estimó en 3.70, mientras que la dominancia de Simpson $\left(\mathrm{S}_{\mathrm{i}}\right)$ se estimó en 0.908 . Las especies con mayor valor de importancia se anotan en el Cuadro 1.

La comunidad media presenta sedimento limo-arcilloso con contenido de materia orgánica promedio de $6.05 \pm .01 \%$. La riqueza de especies es de 46 con una densidad media de $98.6 \pm 28.2 \mathrm{ind} / \mathrm{m}^{2}$. Se enconţó una biomasa media de $58.07 \pm 27.4 \mathrm{mg} / \mathrm{m}^{2}$, sobresaliendo la contribución de la estación ILGOR con $107.65 \mathrm{mg} / \mathrm{m}^{2}$. La diversidad de Shannon-Weaver $\left(\mathrm{H}^{\prime}\right)$ en esta comunidad se estimó en 3.93, mientras que la dominancia de Simpson $\left(\mathrm{S}_{\mathrm{i}}\right)$ se estimó en 0.896. Las especies con mayor valor de importancia se anotan en el Cuadro 1.

CUADRO 1

Valores de importancia de las especies que conforman el 95\% del total de individuos recolectados en cada comunidad

C. Externa

ESPECIE

Boccardia anophtalma (Rioja)

Cossura candida Hartman

Magelona californica Hartman

Mellita longifissa Michelin

Polidora ligni Webster

Paraprionospio pinnata Ehlers

Nassarius luteostoma (Broderip \& Sowerby)

Glycera americana Leidy

$\begin{array}{llllll}\text { CPN } & \text { SCLA } & \text { ELFB } & \text { ESBA } & \text { ILGOR } & \text { ILMON } \\ 18.5 & 20.5 & 36.2 & 31.8 & 56.6 & 42.4 \\ 9.5 & 7.5 & 47.7 & 42.9 & 42.6 & 37.4 \\ 46.8 & 43.2 & 7.9 & 9.8 & 5.25 & 10.5 \\ 54.2 & 66.5 & 0 & 0 & 0 & 0 \\ 0 & 0 & 11.2 & 29.8 & 42.5 & 36.4 \\ 30.7 & 32.7 & 0 & 0 & 0 & 20.1 \\ 24.6 & 35.4 & 8.6 & 7.6 & 0 & 0 \\ 5.4 & 6.6 & 16.9 & 23.8 & 7.4 & 11.6 \\ & & & & & \text { Continúa... }\end{array}$

C. Interna

Continúa... 
...Continuación del Cuadro 1
C. Externa
C. Media
C. Interna

\section{ESPECIE}

Micranellum sp.

Nephtys caecoides Hartman

Armandia sp.

Diastylis sp.

Lumbrineris lagunae Fauchald

Foxiphalus obtusidens Bamard

Allorchestes sp.

Tagelus affinis (Adams)

Phylodoce tuberculosa Kudenov

Eudevenopus metagracilis Barnard

Olivella dama (Wood)

Hyale yaqui Barnard

Tetragrapsus jouyi Rathbun

Rephoxinus sp.

Sinchelidium sp.

Rissolella scolpa Bartsch

$\begin{array}{llllll}\text { CPN } & \text { SCLA } & \text { ELFB } & \begin{array}{c}\text { ESBA } \\ \text { ILGOR }\end{array} & \text { ILMON } \\ 22.4 & 18.6 & 6.2 & 8.1 & 0 & 10.5 \\ 0 & 0 & 0 & 0 & 27.6 & 32.8 \\ 30.3 & 26.7 & 0 & 0 & 0 & 0 \\ 0 & 0 & 28.7 & 26.3 & 0 & 0 \\ 0 & 0 & 27.2 & 27.4 & 0 & 0 \\ 9.6 & 13.4 & 8.6 & 10.3 & 6.8 & 0 \\ 15.2 & 13.8 & 0 & 0 & 7.4 & 7 \\ 0 & 38 & 0 & 0 & 0 & 0 \\ 38 & 0 & 0 & 0 & 0 & 0 \\ 0 & 0 & 8.4 & 11.2 & 0 & 0 \\ 0 & 17 & 0 & 0 & 0 & 0 \\ 6.8 & 4.8 & 0 & 0 & 0 & 0 \\ 0 & 0 & 0 & 11.1 & 0 & 0 \\ 7.4 & 0 & 0 & 0 & 0 & 0 \\ 0 & 0 & 0 & 7.3 & 0 & 0 \\ 0 & 0 & 5.4 & 0 & 0 & 0\end{array}$

La comunidad interna presenta sedimento limoso con contenido promedio de materia orgánica de $7.12 \pm .1 .1 \%$. La riqueza de especies presentes es de 12 , con una densidad media de $28.0 \pm 13.8 \mathrm{inds} / \mathrm{m}^{2}$, la biomasa media es de $1.98 \pm .3 \mathrm{mg} / \mathrm{m}^{2}$. La diversidad de Shannon-Weaver (H') en esta comunidad se estimó en 2.12, mientras que la dominancia de Simpson $\left(\mathrm{S}_{\mathrm{i}}\right)$ se estimó en 2.12. Las especies con mayor valor de importancia se anotan en el Cuadro 1.

\section{DISCUSIÓN}

La textura del sedimento sugiere que la acción del oleaje tiene un efecto mayor en la zona exterior disminuyendo hacia la parte interior del delta. Por otra parte la energía de la marea actúa más intensamente en el interior del delta. Este cambio de energía puede ser la fuerza que determina el cambio de la comunidad, tal como lo sugieren Armonies y Hellwig-Armonies (1987).

La comunidad externa se presenta en sedimento que indica influencia marina y es la única zona con habitación humana, A esto puede atribuirse que los índices comunitarios sean menores que en la comunidad media sin embargo los valores de diversidad, biomasa y estructuración son semejantes a otros sitios, como el Estuario del Río San Lorenzo (Miron y Desrosiers 1990) o la Ría de Foz (Junoy y Véitez 1990).

La comunidad media es la más rica en biomasa y en especies, así como en materia orgánica, aquí el sedimento es más fino. El dominante aparente es el crustáceo Uca princeps debido a que, según se ha reportado para otras especies del género (Montague 1975, Marples 1966), modifica el sedimento por su actividad excavadora haciendo cambios profundos en el ambiente, lo que se refleja en la estructura de la comunidad. Esta dominancia no se refleja en los valores de importancia debido a que los métodos de recolecta usados subestiman la abundancia del cangrejo.

La comunidad interna muestra un empobrecimiento en densidad, biomasa y diversidad con respecto a las comunidades media y externa. Esta comunidad habita una zona muy inestable, sujeta a gran erosión (Cupul-Magaña, 1994) lo que indudablemente está afectando las posibilidades de desarrollo de la comunidad. 


\section{AGRADECIMIENTOS}

Agradecemos al proyecto Delta Camarón de la Universidad Autónoma de Baja California por el apoyo en las salidas de campo y en los análisis granulométrico y de biomasa.

\section{REFERENCIAS}

Armonies, W. \& M. Hellwig-Armonies. 1987. Synoptic patterns of meiofaunal and macrofaunal abundances and specific composition in littoral sediments. Helg. Wiss. Meer. 41: 83-111.

Brian, R.T. \& J.T. Curtis. 1952. The upland conifer-hardwood forest of northern Wisconsin. Ecol. Monogr. 22: 217-234.

Cupul-Magaña, A.L. 1994. Flujos de sedimento en suspención y de nutrientes en la cuenca estuarina del Río Colorado. Tesis de Maestría, Universidad Autónoma de Baja California, Ensenada, México.

Dean, W.E. 1974. Determination of carbonate and organic matter in calcareous sediments and sedimentary rocks by loss ignition: Comparison with other methods. J. Sed. Petrol. 44: 57-68.

Farfán, C. \& S. Alvarez-Borrego. 1992. Zooplankton biomass of the northernmost Gulf of California. Ciencias Marinas 18: 17-36.
Folk, R.L. 1961. Petrology of sedimentary rocks. Hemphill, Austin, Texas. 78p.

Junoy, J. \& J.M. Véitez. 1990. Macrozoonenthic community structure in the Ría de Foz, an intertidal stuary (Galicia, Norwest Spain). Mar. Biol. 107: 329-339.

Marples, T.G. 1966. A radionuclide tracer study of arthropod food chains in Spartina sp. Salt marsh ecosystem. Ecology 47: 270-277.

Martínez-Rojas-Reynoso, M.K. 1990. Distribución espacial y registros circadianos de temperatura, salinidad y oxígeno disuelto en el delta del Río Colorado. Tesis Licenciatura, Universidad Autónoma de Baja California, Ensenada, México.

Miron, G.Y. \& G.L. Desrosiers. 1990. Distribution and population structures of two intertidal estuarine polichaetes in the lower St. Lawrence estuary, with special reference to environmental factors. Mar. Biol. 105: 297-306.

Montague, L.C. 1975. A Natural History of temperate western atlantic fiddler crab (Genus $U c a$ ) with reference of their impact on the salt marsh. Cont. Mar. Sci. 23: 25-55.

Villarreal-Chávez, G. 1992. Algunos aspectos de la biología y ecología de Callinectes arcuatus en la zona estuarina del Río Colorado. Proc. San Diego Soc. Nat. Hist. 10: 1-4. 